CONFORMAL GEOMETRY AND DYNAMICS

An Electronic Journal of the American Mathematical Society

Volume 5, Pages 6-20 (March 30, 2001)

S $1088-4173(01) 00060-1$

\title{
ROTATION ESTIMATES AND SPIRALS
}

\author{
VLADIMIR GUTLYANSKIII AND OLLI MARTIO
}

\begin{abstract}
It is shown that the logarithmic spiral gives the extremum to F. John's angle distortion problem for plane bilipschitz mappings. The problem of factoring spiral-like mappings into a composition of homeomorphisms with smaller isometric distortion is studied. A space counterpart of the Freedman and He theorem is obtained.
\end{abstract}

\section{Introduction And Main Results}

Rotation problems are extensively studied in function theory and its applications to geometry and dynamics. A typical problem from the nonlinear elasticity theory is due to F. John. In 7 he showed that if $f: \mathbb{C} \rightarrow \mathbb{C}$ is an $(1+\varepsilon)$-bilipschitz mapping and if for some $0<a<b$ we have $f(z)=z$ for $|z|>b, f(z)=z e^{i \theta}$ for $|z|<a,|\theta| \leq \pi$, then

$$
|\theta| \leq C(1+\log (b / a)) \varepsilon .
$$

The angle estimate (1.1) follows from the basic stability theorems in [7] for $(1+\varepsilon)$ bilipschitz mappings in the plane. The BMO technique 8 also plays an important role for (1.1).

We show that the proper framework for (1.1) concerns quasiconformal mappings which are more general than bilipschitz mappings. Quasiconformal methods lead to the sharp solution of John's problem. They also provide sharp integral estimates for the rotation angle in terms of the pointwise quasiconformal and isometric distortion coefficients. Before the statements, we recall some notation.

Let $G$ be a domain in the complex plane $\mathbb{C}$. A sense preserving homeomorphism $f: G \rightarrow \mathbb{C}$ is called $Q$-quasiconformal, $Q \geq 1$, if $f \in W_{l o c}^{1,2}(G)$ and if

$$
\left\|f^{\prime}(z)\right\|^{2} \leq Q J_{f}(z) \text { a.e. in } G \text {. }
$$

Here $J_{f}(z)$ is the Jacobian determinant of $f^{\prime}(z)$ and $\left\|f^{\prime}(z)\right\|=\left|f_{z}(z)\right|+\left|f_{\bar{z}}(z)\right|$. We shall employ the pointwise distortion coefficient of $f$ at $z$ defined as

$$
K_{f}(z)=\left\|f^{\prime}(z)\right\|^{2} / J_{f}(z) .
$$

This coefficient is well-defined at the regular points of $f$ in $G$, hence almost everywhere, and we set $K_{f}(z)=1$ at the nonregular points.

Received by the editors March 17, 2000 and, in revised form, January 4, 2001.

2000 Mathematics Subject Classification. Primary 30C62, 30 C65.

The authors thank the Mittag-Leffler Institute for financial support during the fall of the academic year 1999/2000. 
A homeomorphism $f: G \rightarrow \mathbb{C}$ is said to be an $L$-bilipschitz if it satisfies the double inequality

$$
\frac{1}{L}\left|z-z^{\prime}\right| \leq\left|f(z)-f\left(z^{\prime}\right)\right| \leq L\left|z-z^{\prime}\right|
$$

whenever $z, z^{\prime} \in G$. The smallest $L \geq 1$ for which (1.4) holds is called the isometric distortion of $f$. Note that each $L$-bilipschitz mapping $f$ is $L^{2}$-quasiconformal.

Theorem 1.1. Let $f$ be a Q-quasiconformal mapping of the annulus $R(a, b): a \leq$ $|z| \leq b$ such that $f(z)=z$ for $|z|=b, f(z)=z e^{i \theta}$ for $|z|=a$. Then for every continuous nondecreasing convex function $\Phi$,

$$
\int_{R(a, b)} \Phi\left(K_{f}(z)\right) \frac{d m_{z}}{|z|^{2}} \geq \int_{R(a, b)} \Phi\left(K_{f *}(z)\right) \frac{d m_{z}}{|z|^{2}}
$$

where the extremal mapping $f^{*}$ is defined in $R(a, b)$ as

$$
f^{*}(z)=b s_{k}(z / b), \quad k=-\theta / \log (b / a),
$$

with

$$
s_{k}(z)=z e^{i k \log |z|} .
$$

The following result provides a sharp integral estimate for the rotation angle $\theta$ in terms of the pointwise quasiconformal distortion coefficient.

Theorem 1.2. Let $f$ be a $Q$-quasiconformal mapping of the annulus $R(a, b): a \leq$ $|z| \leq b$ such that $f(z)=z$ for $|z|=b, f(z)=z e^{i \theta}$ for $|z|=a$. Then

$$
|\theta|\left(\frac{|\theta|}{2 \log (b / a)}+\sqrt{1+\frac{\theta^{2}}{4 \log ^{2}(b / a)}}\right) \leq \frac{1}{2 \pi} \int_{R(a, b)} \frac{K_{f}(z)-1}{|z|^{2}} d m_{z} .
$$

The estimate (1.8) is sharp and the mapping (1.6) provides the equality.

Indeed, (1.6) and (1.7) imply that

$$
\left|f_{z}^{*}\right|=\sqrt{1+k^{2} / 4}, \quad\left|f_{\bar{z}}^{*}\right|=|k| / 2
$$

where the constant $k$ is defined by (1.6). Hence $J_{f^{*}}=\left|f_{z}^{*}\right|^{2}-\left|f_{\bar{z}}^{*}\right|^{2}=1$ for all $z \in R(a, b)$ and therefore $f^{*}$ is volume preserving. Using formula (1.3) we obtain

$$
K_{f^{*}}(z)=\left(|k| / 2+\sqrt{1+k^{2} / 4}\right)^{2}, \quad k=-\theta / \log (b / a) .
$$

Since

$$
K_{f^{*}}(z)-1=|\theta|(\log (b / a))^{-1}\left(|\theta| / 2 \log (b / a)+\sqrt{1+\theta^{2} / 4 \log ^{2}(b / a)}\right)
$$

and

$$
\frac{1}{2 \pi} \int_{R(a, b)} \frac{d m_{z}}{|z|^{2}}=\log (b / a)
$$

the inequality (1.8) follows from (1.5) if we set $\Phi(t)=t-1$. 
Remark 1.3. By elementary calculation the sharp inequality (1.8) can be written in the equivalent form

$$
|\theta| \leq\left(I^{1 / 2}\left(K_{f}\right)-1 / I^{1 / 2}\left(K_{f}\right)\right) \log (b / a)
$$

where

$$
I\left(K_{f}\right)=\frac{1}{2 \pi \log (b / a)} \int_{R(a, b)} \frac{K_{f}(z)}{|z|^{2}} d m_{z} .
$$

The estimates (1.8) and (1.13) can be viewed as angle-distortion stability results.

Corollary 1.4. Let $f$ be an L-bilipschitz homeomorphism of the annulus $R(a, b)$ such that $f(z)=z$ for $|z|=b, f(z)=z e^{i \theta}$ for $|z|=a$. Then

$$
|\theta| \leq(L-1 / L) \log (b / a) \text {. }
$$

The estimate (1.15) is sharp and the mapping (1.6) provides the equality.

Indeed, since $f$ is also $L^{2}$-quasiconformal, then $K_{f}(z) \leq L^{2}$ a.e. in $R(a, b)$ and hence the inequality (1.8) yields (1.15).

The sharp form of John's inequality follows now immediately, if we replace $L$ in (1.15) by $1+\varepsilon$.

Corollary 1.5. Let $f$ be an $(1+\varepsilon)$-bilipschitz homeomorphism of the annulus $R(a, b): a \leq|z| \leq b$ such that $f(z)=z$ for $|z|=b, f(z)=z e^{i \theta}$ for $|z|=a$. Then

$$
|\theta| \leq \frac{\varepsilon(2+\varepsilon)}{1+\varepsilon} \log (b / a) .
$$

For each $L>1$ the $L$-bilipschitz mapping $s_{k},|k|=L-1 / L$, transforms the radial lines into spirals, infinitely winding about the origin and it is called the logarithmic spiral mapping. The spiral mappings play an important role in applications. F. Gehring employed $s_{k}$ in 3 to solve the well-known Bers's problem on the structure of the universal Teichmüller space and applied the logarithmic spiral mapping in 44 for studying the injectivity problem for local quasi-isometries. F. John [9] used the mapping $s_{k}$ to study the uniqueness of nonlinear elastic equilibrium for prescribed boundary displacements. A solution of the well-known Teichmüller-Wittich-Belinski-Lehto conformal differentiation and regularity problems for quasiconformal mappings in the plane was also done by means of the spiral mapping (see e.g., [11], p. 232, [16], [1], [10]). A similar problem in space has been studied by Yu. Reshetnyak [14].

In Sections 3 and 4 we study the spiral and spiral-like mappings in plane and space and their factoring into homeomorphisms with small isometric distortion.

It is well-known that every $L$-bilipschitz mapping between two one-dimensional intervals can be factored into a composition of homeomorphisms with smaller isometric distortion $\alpha$. Such a composition can be written explicitly and it requires $N<\log _{\alpha} L+1$ factors. In fact, if $f: I \rightarrow I_{1}$ is an $L$-bilipschitz mapping, then $f$ can be factored as $f=f_{2} \circ f_{1}$, where $f_{1}$ is the $\alpha$-bilipschitz mapping defined by

$$
f_{1}(x)=\int_{x_{0}}^{x}\left|f^{\prime}(t)\right|^{\lambda} d t, \quad \lambda=\log _{L} \alpha,
$$

where $x_{0} \in I$ is fixed and $f_{2}$ is the $L / \alpha$-bilipschitz mapping $f \circ f_{1}^{-1}$. 
For $n \geq 2$ it is not known if every bilipschitz mapping can be factored in the above sense. M. Freedman and Z.-Xu. He [2] studied the logarithmic spiral $s_{k}$ and showed that it requires $N \geq|k| /\left(\alpha^{2}-1\right)^{1 / 2}$ factors to be represented as a composition of $\alpha$-bilipschitz mappings. Since $s_{k}$ is also $L^{2}$-quasiconformal, then by the general analytic theory of quasiconformal mappings in the plane, the minimal number of factors of $s_{k}$ with conformal distortion $\leq(1+\varepsilon)$ grows like $2 \log _{1+\varepsilon} k$ when $k$ goes to the infinity. Thus for large $k$ the spiral (1.7) takes more factors to unwind by means of bilipschitz mappings with small isometric distortion than with conformal distortion.

The following statements provide additional information on the factoring of the logarithmic spiral. It seems likely that the Freedman and He estimate for the number of factors can be improved. We study a special set of volume preserving bilipschitz automorphisms of the unit disk $B^{2}$ and show that in this class a better lower bound is obtained.

Let $\theta$ be a locally absolutely continuous real valued function in the interval $(0,1]$ with

$$
\left|\theta^{\prime}(t)\right| \leq \frac{\alpha^{2}-1}{2 \alpha t}, \text { a.e. }
$$

We define the mapping $f: B^{2} \rightarrow B^{2}$ as

$$
f(z)=z e^{i \theta(z \bar{z})}, \quad f(0)=0 .
$$

Clearly $f$ is a homeomorphism of $B^{2}$ which maps each circle $S^{1}(r), 0<r \leq 1$ onto itself. The following lemma shows that $f$ is actually bilipschitz.

Lemma 1.6. The mapping $f$ is a volume preserving $\alpha$-bilipschitz mapping of $B^{2}$.

The class of mappings (1.19) we will denote by $\mathcal{P}$.

Proposition 1.7. Let $s_{k}$ be factored as $s_{k}=f_{N} \circ \cdots \circ f_{1}$, where each $f_{j} \in \mathcal{P}$. Then

$$
N \geq|k| /(\alpha-1 / \alpha)
$$

if $|k| /(\alpha-1 / \alpha)$ is a positive integer and

$$
N \geq[|k| /(\alpha-1 / \alpha)]+1
$$

otherwise. Here $[c]$ stands for the whole part of the real number $c$. These estimates are sharp.

Contrary to the plane, little is known on factoring in space. An affine $L$ bilipschitz mapping of $\mathbb{R}^{n}$ onto $\mathbb{R}^{n}$ can be factored into a composition of $N<$ $\log _{\alpha} L+1$ affine $\alpha$-bilipschitz mappings.

In Section 4 we study the factoring problem for the volume preserving bilipschitz automorphism $S_{k}$ of the unit ball $B^{3}$ in 3 -space defined as

$$
S_{k}(x)=\left(\left(x_{1}+i x_{2}\right) e^{i k \log |x|}, \quad x_{3}\right), \quad S_{k}(0)=0, \quad x=\left(x_{1}, x_{2}, x_{3}\right), \quad k \in \mathbb{R}^{1} .
$$

The mapping (1.22) was introduced in [5] to study the distortion and rotation problems for quasiconformal mappings in space. This mapping can be viewed as a space counterpart of (1.7). The trace of $S_{k}$ in $\mathbb{C}$ gives us again the mapping (1.7).

It turns out that the special class of volume preserving bilipschitz mappings $f: B^{3} \rightarrow B^{3}$ with

$$
\left\langle f(x), e_{3}\right\rangle=\left\langle x, e_{3}\right\rangle \text { and }|f(x)|=|x|
$$


for each $x \in B^{3}$ forms a test class for the composition. Here $e_{1}, e_{2}, e_{3}$ denote the standard unit vectors of $\mathbb{R}^{3}$. In what follows, a volume preserving bilipschitz mapping $f: B^{3} \rightarrow B^{3}$ satisfying (1.23) for all $x \in B^{3}$ will be called the bilipschitz rotation of the unit ball in $\mathbb{R}^{3}$ about the $e_{3}$-axis. A bilipschitz rotation $f$ of $B^{3}$ maps each circle $C(r, t)=\left\{x \in S^{2}(r): x_{3}=t\right\}, 0<r<1,|t|<r$, onto itself. However, $f$ need not map the circles $C\left(r, t_{1}\right)$ and $C\left(r, t_{2}\right)$ onto itself in a similar fashion as the mapping $S_{k}$ does, i.e., $f$ need not be a rotation of $S^{2}(r)$.

Theorem 1.8. Let $S_{k}: B^{3} \rightarrow B^{3}$ be the $(1+|k|)$-bilipschitz mapping defined by (1.22). If $S_{k}$ is factored as $S_{k}=f_{N} \circ f_{N-1} \circ \cdots \circ f_{1}$, where every $f_{j}$ is an $\alpha$-bilipschitz rotation of $B^{3}$, then

$$
N \geq|k| \frac{\sqrt{3 / \pi}}{\left(\alpha^{3}-1\right)^{1 / 2}} .
$$

\section{Proof of Theorem 1.1}

For simplicity and without loss of generality we may assume that $a=q, 0<q<$ 1 , and $b=1$. The test class $\mathcal{M}$ consists of all $Q$-quasiconformal mappings $f$ of the annulus $R(q, 1)$ onto itself such that $f(z)=z$ for $|z|=1, f(z)=z e^{i \theta}$ for $|z|=q$. We will show that

$$
\min _{f \in \mathcal{M}} \int_{R(q, 1)} \Phi\left(K_{f}(z)\right) \frac{d m_{z}}{|z|^{2}}=\int_{R(q, 1)} \Phi\left(K_{f^{*}}(z)\right) \frac{d m_{z}}{|z|^{2}}
$$

for every continuous nondecreasing convex function $\Phi$. The proof is by contradiction. Suppose that there exists a mapping $f \in \mathcal{M}$ such that

$$
\int_{R(q, 1)} \Phi\left(K_{f}(z)\right) \frac{d m_{z}}{|z|^{2}}<\int_{R(q, 1)} \Phi\left(K_{f^{*}}(z)\right) \frac{d m_{z}}{|z|^{2}} .
$$

For fixed $n=1,2, \ldots$, we will introduce the mapping $\varphi(z)$ defined in $R\left(q^{1 / n}, 1\right)$ as

$$
\varphi(z)=z\left(\frac{f\left(z^{n}\right)}{z^{n}}\right)^{1 / n}
$$

and normalized by $\varphi(1)=1$. This mapping is well-defined since $f$ is a sensepreserving homeomorphism of the annulus $R(q, 1)$ onto itself. The boundary conditions of $f$ imply that $\varphi(z)=z$ for $|z|=1$ and $\varphi(z)=z e^{i \theta / n}$ for $|z|=q^{1 / n}$. Considering the conformal covering spaces of the annuli, we see that the mapping $\varphi$ is $n$-fold symmetric with respect to the origin in the sense that

$$
\varphi\left(\varepsilon_{j} z\right)=\varepsilon_{j} \varphi(z)
$$

for every $\varepsilon_{j}=e^{i 2 \pi j / n}, j=1,2, \ldots, n-1$, and is one-to-one in $R\left(q^{1 / n}, 1\right)$. Since $f$ is quasiconformal in $R(q, 1)$, the mapping $\varphi$ is also quasiconformal in $R\left(q^{1 / n}, 1\right)$.

Using $\varphi$ we generate the following sequence of mappings

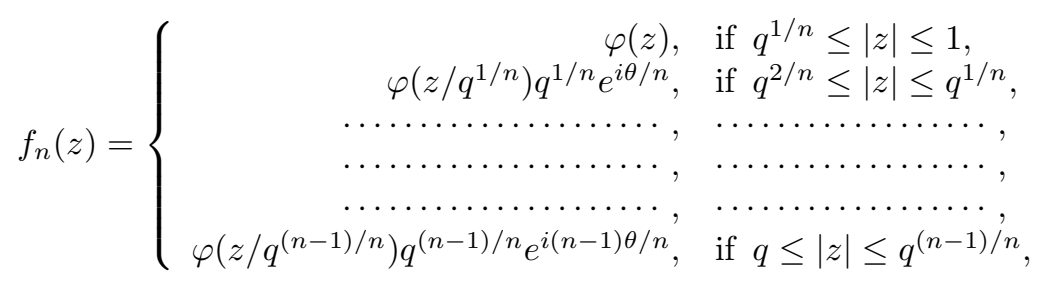


and show that each $f_{n}$ belongs to the set $\mathcal{M}$.

First we note that, by the construction, each $f_{n}$ is quasiconformal in $R(q, 1) \backslash$ $\bigcup_{j=1}^{n-1}\left\{|z|=q^{j / n}\right\}$ and continuous in $R(q, 1)$. Next, since $f_{n}$ maps each circle $|z|=$ $q^{j / n}, j=1, \ldots, n-1$, onto itself, then by the removable singularity principle for quasiconformal mappings (see [11]) $f_{n}$ is quasiconformal in $R(q, 1)$. Finally, the admissible normalization of $f_{n}$ for $|z|=1$ and $|z|=q$ follows from the boundary conditions of the mapping $\varphi$. Thus $f_{n} \in \mathcal{M}$ for all $n=1,2, \ldots$.

On the other hand, we see that $f_{n}\left(q^{j / n} e^{i \omega}\right)=q^{j / n} e^{\omega+j \theta / n}$ for all $n=1,2, \ldots$, $j=1, \ldots, n-1$ and $0 \leq \omega<2 \pi$. It is immediate now that the sequence $f_{n}$ converges uniformly in $R(q, 1)$ to the mapping $f^{*}$ defined by formula (1.6). Next we will make use of the following convergence result proved in [6] (see Theorem 4.1). It states that if $F_{n}: G \rightarrow \mathbb{C}, n=1,2, \ldots$, is a sequence of $Q$-quasiconformal mappings that converges locally uniformly in $G$ to $F$, then

$$
\int_{E} \Phi\left(K_{F}(z)\right) d m_{z} \leq \liminf _{n \rightarrow \infty} \int_{E} \Phi\left(K_{F_{n}}(z)\right) d m_{z}
$$

for every measurable set $E \subset G$ with $m(E)<\infty$ and for every continuous nondecreasing convex function $\Phi$. We will apply this statement to the sequence $f_{n}$ in the following way. First we show that

$$
\int_{R(q, 1)} \Phi\left(K_{f_{n}}(z)\right) \frac{d m_{z}}{|z|^{2}}=\int_{R(q, 1)} \Phi\left(K_{f}(z)\right) \frac{d m_{z}}{|z|^{2}}
$$

for every $n=1,2, \ldots$. To this end observe that the Jacobian determinant of the mapping $g(z)=z^{n}$ satisfies $J_{g}(z)=\left|g^{\prime}(z)\right|^{2}=n^{2}|z|^{2 n-2}$ and hence

$$
\begin{aligned}
& \int_{R\left(q^{1 / n}, 1\right)} \Phi\left(K_{f_{n}}(z)\right) \frac{d m_{z}}{|z|^{2}}=\frac{1}{n^{2}} \int_{R\left(q^{1 / n}, 1\right)} \Phi\left(K_{f}\left(z^{n}\right)\right) \frac{J_{g}(z)}{|z|^{2 n}} d m_{z} \\
= & \frac{1}{n^{2}} \int_{R(q, 1)} \Phi\left(K_{f}(z)\right) N(g, z) \frac{d m_{z}}{|z|^{2}}=\frac{1}{n} \int_{R(q, 1)} \Phi\left(K_{f}(z)\right) \frac{d m_{z}}{|z|^{2}} .
\end{aligned}
$$

Note that the multiplicity $N(g, z)=\operatorname{card}\left(g^{-1}(z) \cap R\left(q^{1 / n}, 1\right)\right)$ of $g$ in $R\left(q^{1 / n}, 1\right)$ satisfies $N(g, z)=n$. By symmetry we also have

$$
\int_{R\left(q^{\frac{j}{n}}, q^{\frac{(j-1)}{n}}\right)} \Phi\left(K_{f_{n}}(z)\right) \frac{d m_{z}}{|z|^{2}}=\frac{1}{n} \int_{R(q, 1)} \Phi\left(K_{f}(z)\right) \frac{d m_{z}}{|z|^{2}}
$$

for $j=1,2, \ldots, n$ and summing up we obtain (2.6).

Now (2.6) yields

$$
\liminf _{n \rightarrow \infty} \int_{R(q, 1)} \Phi\left(K_{f_{n}}(z)\right) \frac{d m_{z}}{|z|^{2}}=\int_{R(q, 1)} \Phi\left(K_{f}(z)\right) \frac{d m_{z}}{|z|^{2}} .
$$

Replacing the sequence $f_{n}$ by the sequence $F_{n}(z)=f_{n}\left(e^{z}\right)$ of quasiconformal mappings defined in the rectangle $\Omega:-\log (1 / q) \leq \operatorname{Re} z \leq 0,0 \leq \operatorname{Im} z \leq 2 \pi$, we see that

$$
\int_{R(q, 1)} \Phi\left(K_{f_{n}}(z)\right) \frac{d m_{z}}{|z|^{2}}=\int_{\Omega} \Phi\left(K_{F_{n}}(z)\right) d m_{z} .
$$


Since $F_{n}(z) \rightarrow F(z)=f^{*}\left(e^{z}\right)$ uniformly in $\Omega$ as $n \rightarrow \infty$, the aforementioned convergence theorem implies that

$$
\liminf _{n \rightarrow \infty} \int_{\Omega} \Phi\left(K_{F_{n}}(z)\right) d m_{z} \geq \int_{\Omega} \Phi\left(K_{F}(z)\right) d m_{z} .
$$

Combining (2.9) and (2.10) with (2.11) and going back to the annulus $R(q, 1)$ we obtain that

$$
\int_{R(q, 1)} \Phi\left(K_{f}(z)\right) \frac{d m_{z}}{|z|^{2}} \geq \int_{\Omega} \Phi\left(K_{F}(z)\right) d m_{z}=\int_{R(q, 1)} \Phi\left(K_{f^{*}}(z)\right) \frac{d m_{z}}{|z|^{2}} .
$$

Formula (2.12) provides a contradiction to the inequality (2.2). This completes the proof of the theorem.

As we have already noted, the sharp inequality (1.15) is a consequence of (1.8) since an $L$-bilipschitz mapping in the plane is $L^{2}$-quasiconformal. On the other hand, if for every $f \in \mathcal{M}$

$$
Q(f)=\underset{R(q, 1)}{\operatorname{ess} \sup } K_{f}(z)
$$

denotes the maximal dilatation of $f$ in $R(q, 1)$, then the inequality (1.15) can be obtained if we show that

$$
\min _{f \in \mathcal{M}} Q(f)=Q\left(f^{*}\right)
$$

with $f^{*}$ as in (1.6). The problem (2.14) is Teichmüller's type extremal problem for the class $\mathcal{M}$ of quasiconformal mappings in the annulus $R(q, 1)$ with given boundary values. It could be studied by the standard methods in the logarithmic plane (see e.g., [13]). Below we give an approach, which does not use any modulus estimates, and could be of independent interest. that

The proof is by contradiction. Suppose that there exists a mapping $f \in \mathcal{M}$ such

$$
Q(f)<Q\left(f^{*}\right) .
$$

Let us again make use of the sequence $f_{n}$ generated by $f$ as in the proof of Theorem 1.1. Then by the well-known semicontinuity property of the maximal dilatation coefficients (see [11, [15]) we arrive at the inequality

$$
Q\left(f^{*}\right) \leq \lim \inf _{n \rightarrow \infty} Q\left(f_{n}\right) .
$$

Since $Q\left(f_{n}\right)=Q(f)$ for all $n=1,2, \ldots$, then (2.16) implies that

$$
Q\left(f^{*}\right) \leq Q(f) .
$$

Formula (2.17) provides a contradiction to the inequality (2.15) and (2.14) follows.

The weighted integral mean in $(1.8)$ is closely related to the study of the wellknown conformal differentiability problem (see e.g., [6], p. 232). For example, if

$$
\frac{1}{2 \pi} \int_{|z|<r} \frac{K_{f}(z)-1}{|z|^{2}} d m_{z} \rightarrow 0 \text { as } r \rightarrow 0
$$

then Theorem 1.1 applied to a quasiconformal mapping $f, f(0)=0$, that rigidly rotates each circle $|z|=r$ implies that $f^{\prime}(0)=R$ where $R$ is a rotation. 


\section{Spiral-Like Maps in Plane}

We first prove Lemma 1.6 Assume that $f$ is defined in the unit disk $B^{2}$ by the formula (1.19) with locally absolute continuous real valued function $\theta(t), t \in(0,1]$, satisfying (1.18). Then by means of the straightforward computation we see that

$$
\left|f_{z}(z)\right|=\left(1+t^{2} \theta^{\prime 2}(t)\right)^{1 / 2}, \quad\left|f_{\bar{z}}(z)\right|=t\left|\theta^{\prime}(t)\right|, \quad t=|z|^{2},
$$

a.e. in $B^{2}$. We note also that the mapping $f$ is volume preserving since $\left|f_{z}(z)\right|^{2}-$ $\left|f_{\bar{z}}(z)\right|^{2}=1$. Hence, (1.18) and (3.1) yield

$$
\left\|f^{\prime}(z)\right\|=\left|f_{\bar{z}}(z)\right|+\left|f_{z}(z)\right| \leq \frac{\alpha^{2}-1}{2 \alpha}+\left(1+\left(\frac{\alpha^{2}-1}{2 \alpha}\right)^{2}\right)^{1 / 2}=\alpha .
$$

Since $f$ is volume preserving, we also have $\ell\left(f^{\prime}(z)\right)=1 /\left\|f^{\prime}(z)\right\| \geq 1 / \alpha$ where $\ell\left(f^{\prime}(z)\right)=\left|f_{z}(z)\right|-\left|f_{\bar{z}}(z)\right|$. Now $B^{2}$ is convex and we conclude from the above estimates that $f$ is $\alpha$-bilipschitz.

Corollary 3.1. Suppose that $\theta(t)$ satisfies the inequality

$$
\left|\theta\left(t_{1}\right)-\theta\left(t_{2}\right)\right| \leq \varepsilon\left|\log t_{1}-\log t_{2}\right| .
$$

Then $f$ defined in $B^{2}$ by (1.19) is an $\alpha$-bilipschitz mapping with $\alpha=\varepsilon+\sqrt{1+\varepsilon^{2}}$. In particular, $f$ is an $(1+\varepsilon)$-bilipschitz mapping.

Proof. Write $\eta(s)=\theta\left(e^{s}\right),-\infty<s<0$. Then the inequality (3.3) yields

$$
\left|\eta\left(s_{1}\right)-\eta\left(s_{2}\right)\right| \leq \varepsilon\left|s_{1}-s_{2}\right| .
$$

The last estimate shows that the function $\eta$ is absolutely continuous in $(-\infty, 0)$ and $\left|\eta^{\prime}(s)\right| \leq \varepsilon$ a.e. Hence $\left|t \theta^{\prime}(t)\right| \leq \varepsilon$ a.e in $(0,1)$. By Lemma 1.6 it follows that $f$ is an $\alpha$-bilipschitz mapping with $\alpha=\varepsilon+\sqrt{1+\varepsilon^{2}}$. The elementary estimate $\varepsilon+\sqrt{1+\varepsilon^{2}} \leq 1+\varepsilon$ gives the last conclusion. For a similar result see [4].

Note that the spiral mapping (1.7) is contained in the class of mappings defined by (1.19) and is generated by the formula (1.19) with $\theta(t)=(k / 2) \log t$. If we set $|k|=L-1 / L$, then $\left|\theta^{\prime}(t)\right|=\left(L^{2}-1\right) / 2 t L$ and by Lemma 1.6 $s_{k}$ is an $L$-bilipschitz mapping.

Proposition 3.2. Let $s_{k}: B^{2} \rightarrow B^{2}$ be the L-bilipschitz mapping defined by (1.7). Then for each $\alpha, 1<\alpha<L$, the mapping $s_{k}$ can be factored into a composition of $\alpha$-bilipschitz mappings. More precisely

$$
s_{k}=\underbrace{s_{v} \circ \cdots \circ s_{v}}_{k / v \text { times }}, \quad v=(\operatorname{sign} k)(\alpha-1 / \alpha)
$$

if $k / v$ is a natural number and otherwise

$$
s_{k}=\underbrace{s_{v} \circ \cdots \circ s_{v}}_{[k / v] \text { times }} \circ s_{u}, \quad u=k-[k / v] v
$$

where $[c]$ stands for the whole part of the real number $c$.

The proof immediately follows from the explicit formula (1.7). 
Proof of Proposition 1.7. Assume that $s_{k}=f_{N} \circ \cdots \circ f_{1}$, where each $f_{j}$ is defined by (1.19) with some $\theta_{j}$ satisfying (1.18). Since $\left|f_{j}(z)\right|=|z|, j=1, \ldots, N$, we see that (1.19) implies that

$$
\frac{k}{2} \log t=\theta_{N}+\cdots+\theta_{1}, \quad t=|z|^{2} .
$$

Differentiating both sides of (3.7) with respect to $t$, we obtain from the estimate (1.18)

$$
\frac{|k|}{2 t}=\left|\theta_{N}^{\prime}(t)+\cdots+\theta_{1}^{\prime}(t)\right| \leq N \frac{\alpha^{2}-1}{2 \alpha t} \text { a.e. }
$$

and thus

$$
N \geq \frac{|k|}{\alpha-1 / \alpha} .
$$

Assume first that $|k| /(\alpha-1 / \alpha)$ is a natural number $N$. Then the sign of equality in (3.8) holds if and only if

$$
\theta_{j}^{\prime}(t)=(\operatorname{sign} k) \frac{\alpha^{2}-1}{2 \alpha t}
$$

a.e. for all $j=1, \ldots, N$. This yields $\theta_{j}(t)=v \log |z|$ with $v=(\operatorname{sign} k)(\alpha-1 / \alpha)$ and hence

$$
f_{j}(z)=z e^{i v \log |z|}=s_{v}(z)
$$

for all $j=1, \ldots, N$. Thus, in this case the optimal composition is of the form $s_{k}=\overbrace{s_{v} \circ \cdots \circ s_{v}}^{N \text { times }}$ with $N=|k| /(\alpha-1 / \alpha)$. The case when $k / v$ is fractional can be handled similarly.

\section{Factoring in Space}

We use in $\mathbb{R}^{3}$ the spherical coordinates $r, \omega, \beta$ as follows: $\beta$ stands for an angle between the radius vector and $e_{3}$; the $e_{1} e_{2}$-plane is identified with the complex plane $\mathbb{C}$, so that $z=x_{1}+i x_{2}=r e^{i \omega} \sin \beta$. We denote by $S^{2}$ the unit sphere in $\mathbb{R}^{3}$ centered at the origin.

The following technical lemma is a space counterpart of the corresponding Gehring's result [4] (see also Corollary 3.1).

Lemma 4.1. Suppose that $\theta(t)$ is a real valued function defined in $(0, \infty)$ such that

$$
\left|\theta\left(t_{1}\right)-\theta\left(t_{2}\right)\right| \leq \varepsilon\left|\log t_{1}-\log t_{2}\right| .
$$

If $f$ is defined in $\mathbb{R}^{3}$ as

$$
f(x)=\left(z e^{i \theta(|x|)}, x_{3}\right), \quad f(0)=0,
$$

then $f$ is an $(1+\varepsilon)$-bilipschitz mapping in $\mathbb{R}^{3}$.

Corollary 4.2. The mapping $S_{k}$ defined in $\mathbb{R}^{3}$ by the formula (1.22) is an $(1+|k|)$ bilipschitz mapping. 
Proof of Lemma 4.1. Let $x=\left(x_{1}, x_{2}, x_{3}\right), y=\left(y_{1}, y_{2}, y_{3}\right) \in \mathbb{R}^{3}$ be distinct points such that $|x| \leq|y|$. We set $z=x_{1}+i x_{2}, w=y_{1}+i y_{2}$, and first assume that $z \neq 0$. Then

$$
\begin{aligned}
|f(x)-f(y)|^{2} & =\left|z e^{i \theta(|x|)}-w e^{i \theta(|y|)}\right|^{2}+\left(x_{3}-y_{3}\right)^{2} \\
& =\left|(z-w) e^{i \theta(|y|)}+z\left(e^{i \theta(|x|)}-e^{i \theta(|y|)}\right)\right|^{2}+\left(x_{3}-y_{3}\right)^{2} \\
& \leq\left(|z-w|+|z|\left|e^{i \theta(|x|)}-e^{i \theta(|y|)}\right|\right)^{2}+\left(x_{3}-y_{3}\right)^{2} .
\end{aligned}
$$

Since

$$
\left|e^{i \theta(|x|)}-e^{i \theta(|y|)}\right| \leq|\theta(|x|)-\theta(|y|)| \leq \varepsilon \log \frac{|y|}{|x|}
$$

and

$$
\log \frac{|y|}{|x|} \leq \frac{|y|}{|x|}-1 \leq \frac{|x-y|}{|x|} \leq \frac{|x-y|}{|z|}
$$

we see that

$$
|z|\left|e^{i \theta(|x|)}-e^{i \theta(|y|)}\right| \leq \varepsilon|x-y| .
$$

Now (4.2) and (4.3) yield

$$
\begin{aligned}
|f(x)-f(y)|^{2} & \leq(|z-w|+\varepsilon|x-y|)^{2}+\left(x_{3}-y_{3}\right)^{2} \\
& =|x-y|^{2}+2 \varepsilon|z-w||x-y|+\varepsilon^{2}|x-y|^{2} \leq(1+\varepsilon)^{2}|x-y|^{2}
\end{aligned}
$$

and we arrive at the inequality

$$
|f(x)-f(y)| \leq(1+\varepsilon)|x-y| .
$$

If $z=0$, then

$$
\begin{aligned}
|f(x)-f(y)|^{2}= & |w|^{2}+\left(x_{3}-y_{3}\right)^{2} \leq(|z-w|+\varepsilon|x-y|)^{2} \\
& +\left(x_{3}-y_{3}\right)^{2} \leq(1+\varepsilon)^{2}|x-y|^{2}
\end{aligned}
$$

and we again obtain the inequality (4.4). In order to complete the proof, we note that the inverse mapping $f^{-1}$ is given by (4.1) with $-\theta$ in place of $\theta$ and then to apply (4.4) to the inverse mapping.

Let $\gamma$ be a rectifiable curve in $\mathbb{R}^{n} \backslash\{0\}$ and let $\eta(t):[a, b] \rightarrow \mathbb{R}^{n}$ be its absolutely continuous parametric representation. We call the quantity

$$
\delta_{\gamma}=\int_{a}^{b}\left|\frac{\left|\eta^{\prime}(t)\right|^{2}-|\eta(t)|^{\prime 2}}{|\eta(t)|^{2}}\right|^{\frac{1}{2}} d t
$$

the total rotation of the curve $\gamma$. Note that $\delta_{\gamma}=0$ if and only if $\gamma$ is a radial segment. The integrand in (4.5) is the differential of the angular distance between points $\eta(t)$ and $\eta(t+d t)$. Thus, $\delta_{\gamma}$ can be considered as a space counterpart of the total variation for a continuous branch of $\arg \eta(t)$.

Next, let $f$ be a quasiconformal rotation of the spherical annulus $R(a, b): 0<$ $a<|x|<b$ in $\mathbb{R}^{3}$ onto itself. Given a unit vector $\nu=\left(e^{i \omega} \sin \beta, \cos \beta\right)$ on $S^{2}$ and the radial segment $\gamma_{a b}^{\nu}=t \nu, a \leq t \leq b$, the curve $\gamma=f\left(\gamma_{a b}^{\nu}\right)$ can be parameterized in the spherical coordinates as

$$
\eta(t, \omega, \beta)=\left(t e^{i \Omega(t, \omega, \beta)} \sin \beta, t \cos \beta\right), a \leq t \leq b .
$$


For (4.6) it is enough to observe that $|\eta(t, \omega, \beta)|=t$ for all $\omega$ and $\beta$ since $f$ maps each sphere $S^{2}(t)$ onto itself and the rotation assumption gives

$$
\left\langle f(t \nu), e_{3}\right\rangle=\left\langle t \nu, e_{3}\right\rangle=t \cos \beta .
$$

For every fixed $\beta, 0 \leq \beta \leq \pi$, we introduce the quantity

$$
\mu_{\beta}(f)=\max _{0 \leq \omega \leq 2 \pi}\left|\int_{a}^{b} d \Omega(t, \omega, \beta)\right|
$$

and call it the maximal twist of the corresponding radial segments $\gamma_{a b}^{\nu}$ under the mapping $f$ (cf. [2]). This last quantity does not depend on the particular choice of a continuous branch of the $\Omega(t, \omega, \beta)$ and hence it is well defined.

Lemma 4.3. Let $f_{1}, f_{2}$ be quasiconformal rotations of the spherical annulus $R(a, b)$ in $\mathbb{R}^{3}$ onto itself. Then

$$
\mu_{\beta}\left(f_{1} \circ f_{2}\right) \leq \mu_{\beta}\left(f_{1}\right)+\mu_{\beta}\left(f_{2}\right)+2 \pi .
$$

Proof. Since a composition of two quasiconformal rotations is again a mapping with the same properties, then the curve $f_{1} \circ f_{2}(t \nu), a \leq t \leq b, \nu \in S^{2}$, has the following parametric representation

$$
\eta(t, \omega, \beta)=\left(t e^{\Omega_{1}\left(t, \Omega_{2}(t, \omega, \beta), \beta\right)} \sin \beta, t \cos \beta\right) .
$$

Hence, by formula 4.8

$$
\mu_{\beta}\left(f_{1} \circ f_{2}\right)=\max _{0 \leq \omega \leq 2 \pi}\left|\Omega_{1}\left(b, \Omega_{2}(b, \omega, \beta), \beta\right)-\Omega_{1}\left(a, \Omega_{2}(a, \omega, \beta), \beta\right)\right| .
$$

Since

$$
\begin{aligned}
\left|\Omega_{1}\left(b, \Omega_{2}(b, \omega, \beta), \beta\right)-\Omega_{1}\left(a, \Omega_{2}(a, \omega, \beta), \beta\right)\right| \\
\leq\left|\Omega_{1}\left(b, \Omega_{2}(b, \omega, \beta), \beta\right)-\Omega_{1}\left(a, \Omega_{2}(b, \omega, \beta), \beta\right)\right| \\
\quad+\left|\Omega_{1}\left(a, \Omega_{2}(b, \omega, \beta), \beta\right)-\Omega_{1}\left(a, \Omega_{2}(a, \omega, \beta), \beta\right)\right|,
\end{aligned}
$$

we see that

$$
\mu_{\beta}\left(f_{1} \circ f_{2}\right) \leq \mu_{\beta}\left(f_{1}\right)+\max _{\omega}\left|\Omega_{1}\left(a, \Omega_{2}(b, \omega, \beta), \beta\right)-\Omega_{1}\left(a, \Omega_{2}(a, \omega, \beta), \beta\right)\right| .
$$

Next, since $\Omega_{1}(a, \cdot, \beta)$ is a monotonically increasing function satisfying the condition $\Omega_{1}(a, \omega+2 \pi, \beta)=\Omega(a, \omega, \beta)+2 \pi$ for each $\omega$, then $\left|\Omega_{1}\left(a, \omega_{1}, \beta\right)-\Omega_{1}\left(a, \omega_{2}, \beta\right)\right| \leq$ $\left|\omega_{1}-\omega_{2}\right|+2 \pi$ and therefore

$$
\begin{gathered}
\left|\Omega_{1}\left(a, \Omega_{2}(b, \omega, \beta), \beta\right)-\Omega_{1}\left(a, \Omega_{2}(a, \omega, \beta), \beta\right)\right| \\
\leq\left|\Omega_{2}(b, \omega, \beta)-\Omega_{2}(a, \omega, \beta)\right|+2 \pi .
\end{gathered}
$$

Combining (4.11)-(4.14) we obtain (4.9) and the proof is complete.

Lemma 4.4. Let $f$ be a quasiconformal rotation of the spherical annulus $R(a, b)$ in $\mathbb{R}^{3}$ onto itself. Then

$$
\int_{S^{2}} \delta_{f\left(\gamma_{a b}^{\nu}\right)}^{2} d m(\nu) \leq \frac{2 \log (b / a)}{3} \int_{R(a, b)} \frac{\left\|f^{\prime}(x)\right\|^{3}-1}{|x|^{3}} d x
$$

where $\left\|f^{\prime}(x)\right\|=\sup \left\{\left|f^{\prime}(x) h\right|:|h|=1\right\}$. 
Proof. For a rectifiable curve $\gamma \subset \mathbb{R}^{3} \backslash\{0\}$ we define its quasihyperbolic length (see e.g., [12, [17], p. 33) as

$$
\ell(\gamma)=\int_{\gamma} \frac{|d x|}{|x|} .
$$

Fix a radial segment $\{t \nu: a \leq t \leq b\}, \nu \in S^{2}$, in $R(a, b)$. We shall estimate the quasihyperbolic length of its image under the mapping $f$. Since $f$ is quasiconformal and volume preserving, $f$ is bilipschitz and thus $t \mapsto f(t \nu)$ provides an absolute continuous parameterization for the image curve. The rotation assumption implies that $|f(t \nu)|=t$ for all $\nu \in S^{3}$. Thus

$$
\ell\left(f\left(\gamma_{a b}^{\nu}\right)\right)=\int_{a}^{b}\left|\frac{d}{d t}(f(t \nu))\right| \frac{d t}{t} \leq \int_{a}^{b}\left\|f^{\prime}(t \nu)\right\| \frac{d t}{t} .
$$

Using Hölder's inequality we get that

$$
\int_{a}^{b}\left\|f^{\prime}(t \nu)\right\| \frac{d t}{t} \leq\left(\int_{a}^{b}\left\|f^{\prime}(t \nu)\right\|^{3} \frac{d t}{t}\right)^{1 / 3} \log ^{2 / 3}(b / a) .
$$

Therefore

$$
\ell^{3}\left(f\left(\gamma_{a b}^{\nu}\right)\right) \leq \log ^{2}(b / a) \int_{a}^{b}\left\|f^{\prime}(t \nu)\right\|^{3} \frac{d t}{t} .
$$

Now (4.16) holds for a.e. $\nu \in S^{2}$ and we integrate both sides of (4.16) over $S^{2}$. The Fubini theorem yields

$$
\int_{S^{2}} \ell^{3}\left(f\left(\gamma_{a b}^{\nu}\right)\right) d m(\nu) \leq \log ^{2}(b / a) \int_{R(a, b)} \frac{\left\|f^{\prime}(x)\right\|^{3}}{|x|^{3}} d x .
$$

This leads to (4.15) as follows. Write $\eta(t)=f(t \nu), a \leq t \leq b$. Then

$$
\ell\left(f\left(\gamma_{a b}^{\nu}\right)\right)=\int_{a}^{b} \frac{\left|\eta^{\prime}(t)\right|}{|\eta(t)|} d t=\int_{a}^{b}\left(\frac{\left|\eta^{\prime}(t)\right|^{2}-|\eta(t)|^{2}}{|\eta(t)|^{2}}+\frac{|\eta(t)|^{\prime 2}}{|\eta(t)|^{2}}\right)^{\frac{1}{2}} d t
$$

and since

$$
\left(\int_{a}^{b} \varphi(t) d t\right)^{2}+\left(\int_{a}^{b} g(t) d t\right)^{2} \leq\left(\int_{a}^{b}\left(\varphi^{2}(x)+g^{2}(t)\right)^{\frac{1}{2}} d t\right)^{2}
$$

with

$$
\varphi(t)=\left|\frac{\left|\eta^{\prime}(t)\right|^{2}-|\eta(t)|^{2}}{|\eta(t)|^{2}}\right|^{\frac{1}{2}}, \quad g(t)=\frac{|\eta(t)|^{\prime}}{|\eta(t)|}
$$

we obtain

$$
\ell^{2}\left(f\left(\gamma_{a b}^{\nu}\right)\right) \geq\left(\int_{a}^{b}\left|\frac{\left|\eta^{\prime}(t)\right|^{2}-|\eta(t)|^{\prime 2}}{|\eta(t)|^{2}}\right|^{\frac{1}{2}} d t\right)^{2}+\left(\int_{a}^{b} \frac{|\eta(t)|^{\prime}}{|\eta(t)|} d t\right)^{2} .
$$


Now the first integral in (4.20) is $\delta_{f\left(\gamma_{a b}^{\nu}\right)}$ and

$$
\int_{a}^{b} \frac{|\eta(t)|^{\prime}}{|\eta(t)|} d t=\log (b / a)
$$

and hence

$$
\ell\left(f\left(\gamma_{a b}^{\nu}\right)\right) \geq\left(\delta_{f\left(\gamma_{a b}^{\nu}\right)}^{2}+\log ^{2}(b / a)\right)^{1 / 2} .
$$

Combining these with 4.17) we get

$$
\begin{gathered}
\int_{S^{2}}\left(\left[\delta_{f\left(\gamma_{a b}^{\nu}\right)}^{2}+\log ^{2}(b / a)\right]^{3 / 2}-\log ^{3}(b / a)\right) d m(\nu) \\
\leq \log ^{2}(b / a) \int_{R(a, b)} \frac{\left\|f^{\prime}(x)\right\|^{3}-1}{|x|^{3}} d x .
\end{gathered}
$$

The proof now follows from the elementary inequality

$$
\left[\delta_{f\left(\gamma_{a b}^{\nu}\right)}^{2}+\log ^{2}(b / a)\right]^{3 / 2}-\log ^{3}(b / a) \geq \frac{3 \log (b / a)}{2} \delta_{f\left(\gamma_{a b}^{\nu}\right)}^{2} .
$$

Lemma 4.5. Let $f$ be a quasiconformal rotation of the spherical annulus $R(a, b)$ in $\mathbb{R}^{3}$ onto itself. If $\mu_{\beta}(f)>2 \pi$ for each $0 \leq \beta \leq \pi$, then

$$
\int_{0}^{\pi}\left(\mu_{\beta}(f)-2 \pi\right)^{2} \sin ^{2} \beta d \beta \leq \frac{\log (b / a)}{3 \pi} \int_{R(a, b)} \frac{\left\|f^{\prime}(x)\right\|^{3}-1}{|x|^{3}} d x .
$$

Proof. Since the curve $f\left(\gamma_{a b}^{\nu}\right)$ has the parametric representation of the form (4.6), formula (4.5) yields

$$
\delta_{f\left(\gamma_{a b}^{\nu}\right)}=\int_{a}^{b}|d \Omega(t, \omega, \beta)| \sin \beta d t
$$

and hence

$$
\delta_{f\left(\gamma_{a b}^{\nu}\right)} \geq|\Omega(a, \omega, \beta)-\Omega(b, \omega, \beta)| \sin \beta .
$$

Let

$$
\mu_{\beta}(f)=\max _{\omega}|\Omega(a, \omega, \beta)-\Omega(b, \omega, \beta)|>2 \pi
$$

for every fixed $\beta, 0<\beta<\pi$. We will show that (4.24) implies the inequality

$$
|\Omega(a, \omega, \beta)-\Omega(b, \omega, \beta)| \geq \mu_{\beta}(f)-2 \pi
$$

for every $\omega$ and all $0 \leq \beta \leq \pi$.

Since $\Omega(t, \cdot, \beta)$ is a continuous monotonically increasing function satisfying $\Omega(t, \omega \pm 2 \pi, \beta)=\Omega(t, \omega, \beta) \pm 2 \pi$, we may assume that

$$
\mu_{\beta}(f)=|\Omega(a, 0, \beta)-\Omega(b, 0, \beta)| .
$$

Next, if $\Omega(a, 0, \beta)>\Omega(b, 0, \beta)$, then we get

$$
\Omega(a, \omega, \beta)-\Omega(b, \omega, \beta) \geq \Omega(a, 0, \beta)-\Omega(b, 2 \pi, \beta)=\mu_{\beta}(f)-2 \pi
$$


whenever $0 \leq \omega \leq 2 \pi$. If $\Omega(b, 0, \beta)>\Omega(a, 0, \beta)$, then

$$
\Omega(b, \omega, \beta)-\Omega(a, \omega, \beta) \geq \Omega(b, 0, \beta)-\Omega(a, 2 \pi, \beta)=\mu_{\beta}(f)-2 \pi
$$

whenever $0 \leq \omega \leq 2 \pi$. Hence the inequality 4.25) follows.

Combining 4.25) and 4.23), we obtain

$$
\delta_{f\left(\gamma_{a b}^{\nu}\right)} \geq\left(\mu_{\beta}(f)-2 \pi\right) \sin \beta .
$$

Inserting (4.26) in (4.15), we arrive at the inequality (4.22) and thus complete the proof.

Proof of Theorem 1.8. Let $S_{k}(x)$ be factored as $S_{k}=f_{N} \circ f_{N-1} \circ \cdots \circ f_{1}$, where each $f_{j}$ is an $\alpha$-bilipschitz rotation of $\mathbb{R}^{3}$. Then, by Lemma 4.3

$$
\mu_{\beta}\left(S_{k}\right) \leq \sum_{j=1}^{N} \mu_{\beta}\left(f_{j}\right)+2 \pi(N-1) .
$$

Using Lemma 4.5 we see that for all $r, 0<r<1$,

$$
\begin{gathered}
\int_{0}^{\pi}\left(\mu_{\beta}\left(f_{j}\right)-2 \pi\right) \sin \beta d \beta \leq\left(\pi \int_{0}^{\pi}\left(\mu_{\beta}\left(f_{j}\right)-2 \pi\right)^{2} \sin ^{2} \beta d \beta\right)^{1 / 2} \\
\leq\left(\frac{\log (1 / r)}{3} \int_{R(r, 1)} \frac{\left\|f_{j}^{\prime}(x)\right\|^{3}-1}{|x|^{3}} d x\right)^{1 / 2} .
\end{gathered}
$$

Since

$$
\int_{0}^{\pi} \mu_{\beta}\left(S_{k}\right) \sin \beta d \beta=2|k| \log (1 / r)
$$

(4.27) and (4.28) yield the following inequality

$$
2|k| \log (1 / r) \leq \sum_{j=1}^{N}\left(\frac{\log (1 / r)}{3} \int_{R(r, 1)} \frac{\left\|f_{j}^{\prime}(x)\right\|^{3}-1}{|x|^{3}} d x\right)^{1 / 2}+4 \pi(2 N-1) .
$$

Since all the mappings $f_{j}(x)$ are $\alpha$-bilipschitz, $\left\|f_{j}^{\prime}(x)\right\| \leq \alpha, j=1, \ldots, N$. Inserting the last estimate in 4.30, we obtain

$$
2|k| \log (1 / r) \leq \sum_{j=1}^{N}\left(\frac{4 \pi \log ^{2}(1 / r)}{3}\left(\alpha^{3}-1\right)\right)^{1 / 2}+4 \pi(2 N-1) .
$$

Dividing both parts of the inequality (4.31) by $\log (1 / r)$ and letting $r \rightarrow 0$, we have

$$
|k| \leq N \sqrt{\pi / 3}\left(\alpha^{3}-1\right)^{1 / 2}
$$

and hence (1.24) follows. 


\section{REFERENCES}

[1] Belinskir, P.P., General properties of quasiconformal mappings, Nauka, Novosibirsk, 1974. (Russian) MR 53:11054

[2] Freedman, M.H. and He, Z-Xu., Factoring the logarithmic spiral, Invent. Math., 92 (1988), 129-138. MR 89j:30026

[3] Genring, F., Spirals and the universal Teichmüller space, Acta Math., 141 (1978), 99-113. MR 58:17076]

[4] Gehring, F.W., Injectivity of local quasi-isometries, Comment. Math. Helvetici, 57 (1982), 202-220. MR 84b:30018

[5] Gutlyanskit, V., Martio, O., Vuorinen, M., Rotation in space, University of Helsinki, Department of Mathematics, Preprint 215, (1999), 23 pp.

[6] Gutlyanskit, V.Ya., Martio, O., Ryazanov, V.I., Vuorinen, M., On convergence theorems for space quasiregular mappings, Forum Math., V. 10 (1998), 353-375. MR 99f:30035

[7] John, F., Rotation and strain, Comm. Pure Appl. Math., 14 (1961), 391-413. MR 25:1672

[8] John, F., Nirenberg, L., On functions of bounded mean oscillation, Comm. Pure Appl. Math., 14 (1961), 415-426. MR 24:A1348

[9] John, F., Uniqueness of non-linear elastic equilibrium for prescribed boundary displacements and sufficiently small strains, Comm. Pure Appl. Math., 25, (1972), 617-634. MR 47:3857

[10] Lehto, O., On the differentiability of quasiconformal mappings with prescribed complex dilatation, Ann. Acad. Sci. Fenn. A I 275 (1960), 1-28. MR 23:A3260

[11] Lehto, O., Virtanen, K., Quasiconformal Mappings in the Plane, Second ed., Translated from the German by K. W. Lucas. Die Grundlehren der mathematischen Wissenschaften, Springer-Verlag, New York-Heidelberg, 1973. MR 49:9202

[12] Martin, G., Osgood, B.G., The quasihyperbolic metric and the associated estimates on the hyperbolic metric, J. Analyse Math., 47 (1986), 37-53. MR 88e:30060

[13] Reich, E., Strebel, K., Extremal quasiconformal mappings with given boundary values, In: Contribution to Analysis. A collection of papers dedicated to Lipman Bers. Academic Press, New-York-London, 1974, 375-391. MR 50:13511

[14] Reshetnyak, Yu. G., Stability theorems in geometry and analysis, Nauka, Sibirskoe otdelenie, Novosibirsk, (1982). (Russian) MR 96i:30016

[15] Strebel, K., Ein Konvergenzsatz für Folgen quasikonformer Abbildungen, Comment. Math. Helv., 44 (1969), 469-475. MR 40:7444

[16] Teichmüller, O., Untersuchungen über konforme und quasikonforme Abbildung, Deutsche Math. 3 (1938), 621-678.

[17] Vuorinen, M., Conformal Geometry and Quasiregular Mappings, Lecture Notes in Math. 1319 (1988), Springer-Verlag, Berlin-New York. MR 89k:30021

Institute of Applied Mathematics and Mechanics, NAS of Ukraine, Ul. Roze LuxemBURG 74, 340114, Donetsk, Ukraine

E-mail address: gut@iamm.ac.donetsk.ua

Department of Mathematics, P. O. Box 4 (Yliopistonkatu 5), Fin-00014 University of Helsinki, Finland

E-mail address: martio@cc.helsinki.fi 